Meijers, Eduard Maurits, „L'Université d'Orléans au XIII" siècle“, in: Meijers, E. M., Études d'Histoire du Droit III. Le droit romain au Moyen Âge. 1. L'enseignement du droit dans trois universités du XIII ${ }^{e}$ siècle. Histoire des sources, hg. von Robert Feenstra / Herman F. W. D. Fischer, Leiden 1959.

\title{
Überlieferung:
}

Handschriften:

Aus Feenstra 1972:

Graz, UB, 117, um 1300, fol. 1-32v (Fassung b).

Leipzig, UB, 911, 14. Jh., fol. 1-46v (Fragment von Fassung a).

Vaticano, BAV, Borgh. 291, 14. Jh. Praescriptio: Incipiunt casus institucionum (Fassung a).

Feenstra 1976 verzeichnet eine weitere Handschrift, die ihm von Peter Weimar mitgeteilt worden war:

Toledo, Archivo y Biblioteca Capitular, 39-27, 14. Jh., fol. 1r-97r.

Frühdrucke:

[Paris oder Rouen: Drucker des Breviarium Rothomagense, um 1480]. GW 11709.

[Lyon: Johann Siber, um 1487]. GW 11710.

[Straßburg: Johann Prüss, um 1488]. GW 11711, HC 4665.

[Lyon: Drucker von Guido de Cumis, um 1490]. GW 11712, C 1488.

[Lyon: Johann Siber, um 1491]. GW 11713, H 4664.

[Freiburg: Kilian Fischer, um 1494]. GW 11714, H 4663.

[Straßburg: Georg Husner, um 1497]. GW 11715, HC 4666.

Feenstra (1972, 248-249) registriert ferner folgende Frühdrucke (nicht eingesehen):

Casus longi super Institutis, Lyon: Gueynard, 1506.

Mit demselben Titel: Lyon: Haguetan, 1508; Lyon 1509; Paris 1509; Lyon: de Benedictis 1509; Lyon 1511; [Lyon] 1511; Lyon 1513; Lyon 1514: Jacques Mareschal; Torino 1514; Lyon 1515: Jacques Mareschal.

\section{R23. Anonymus Franciscanus}

Dyalogus sanctorum fratrum Minorum

Dialogus de vitis sanctorum fratrum Minorum

Rep.: Mohan 478*, Sbaralea mmmdxar.

Studie: Kapitel 2.4 .

Prolog: Incipit: In nomine Patris et Filii et Spiritus Sancti. Amen. Venerabilium gesta patrum dignosque memoria titulos antiquorum studio pietatis annalibus commendare... Explicit:... sed divinae capax gratiae fides adsit credula disciplinae. 
Incipit: Narrator: Multifarie multisque modis olim Deus immensae potentiam maiestatis et divitias gloriae sue manifestans...

Explicit:... non indigne feram me quencumque repperisse consortem.

Autor: Da das Werk auf Geheiß des Ordensgenerals Crescentius von Jesi geschrieben wurde, dürfte der Autor ein Franziskaner aus seinem Umfeld sein. Gamboso (20-26) erwähnt als mögliche Autoren Bonaventura von Iseo oder Marco von Montefeltro. Delormes Zuschreibung an Thomas Tuscus OFM (Tommasso da Pavia, $† 1284$ ), Autor einer Chronik und einer Predigtsammlung (vgl. Ciccarelli), ist wenig überzeugend (Delorme, XI-XV). Sie beruht auf sprachlichen Parallelen zwischen dem Werk des Thomas und dem Dialogus. Doch handelt es sich dabei zumeist um gängige Redewendungen oder zufällige Übereinstimmungen (vgl. Gamboso, 25).

Datierung: Der Autor sagt in Dial. 1, 6, er habe dieses Werk im Auftrag des Ordensgenerals Crescentius von Jesi geschrieben:...obedientia reverendi patris ministri generalis, fratris scilicet Crescentii, praeceptrice... Dessen Amtszeit dauerte vom 4.10.1244 bis 13.7.1247.

Inhalt: Gespräch zwischen Narrator und Auditor. Das zentrale Thema sind die Wunder, die von Franziskanern bewirkt wurden. Der Narrator reiht die Wundererzählungen aneinander, ohne sie miteinander in Beziehung zu bringen. Der Auditor unterbricht ihn bisweilen mit Fragen, die sich auf die theologischen oder doktrinalen Aspekte des Wunders beziehen. Besprochen werden z.B. die Bedeutung des Wassers, das aus dem Grab eines verstorbenen Fraters fließt; der Grund, warum einige Menschen krank oder missgebildet geboren werden; die Art, wie die Teufel die Besessenen beherrschen; die Tatsache, dass die meisten Wundertäter einfache, ungebildete Fratres sind. Der Narrator stützt sich für seine Erklärungen vor allem auf die Bibel. Manchmal werden seine Ausführungen mit einem Redebeitrag des Auditors abgeschlossen, der ihn dazu auffordert, weitere Wunder zu erzählen. In anderen Fällen wechselt der Narrator selbst von der Auslegung zur Erzählung.

Thematisch ist es möglich, eine Struktur zu erkennen. Zuerst konzentriert sich das Gespräch auf Antonius von Padua. Der Narrator beginnt mit einer kurzen Biographie, die vor allem das monastische Leben, die Predigertätigkeit und den Tod des Antonius schildert. Es folgt die Schilderung von ca. vierzig Wundern, fast ausschließlich Heilungen, nach der Art der Krankheit geordnet: Missbildungen, Blindheit, Taubheit, Stummheit, Epilepsie. Dann werden Auferweckungen von 
Toten geschildert, weitere Wunder unterschiedlicher Art und die Strafen, die auf Beleidigungen des Heiligen, auf Verwünschungen oder gedankenloses Reden folgen.

An zweiter Stelle werden die Wunder des Frater Benevenutus de Eugubio besprochen. Sie beginnen mit einer knappen Skizze seines monastischen Lebens. Es folgt eine Abschrift des Briefes, mit dem der Papst eine Untersuchung der Wunder anordnet. Die darauffolgenden Erzählungen beginnen mit dem Namen und Herkunftsort des Zeugen und Wendungen wie iuratus deposit, iusiurando dixit $\mathrm{u}$. Ä. Sie sind nach der Art des Wunders geordnet, zuerst Auferweckung von Toten, dann Austreibungen von Teufeln und Heilungen von Krankheiten, in der Reihenfolge: Missbildungen, Blindheit, Taubheit. Am Ende finden sich einige Heilungen verschiedener Krankheiten.

An dritter Stelle erzählt der Narrator über Frater Ambrosius de Massana, in einer ähnlichen Reihenfolge wie bei Frater Benevenutus: biographische Skizze, Brief des Papstes, Aussagen der Zeugen. Die Wunder sind wieder nach ihrer Art geordnet: Heilungen von Missbildungen und Epilepsie, Austreibungen von Teufeln, Heilung von Geschwüren und von verschiedenen Krankheiten, Wiedererweckung von Toten.

In einem vierten Abschnitt werden einzelne Wunder von mehreren Fratres erzählt. Sie sind nach dem jeweiligen Wundertäter geordnet: Rogerius de Tuderto, Paulus de Marchia, Hermannus de Fulgineo, Martinus, Dominicus de Sancto Marino, Bernardus de Massa, Petrus de Monte Ulmonis, Leonardus de Fulgineo oder de Piperno, Jacobus de Assissio, Petrus de Trano, Adam Rufus, Guillelmus de Cordella.

Das Werk endet mit der Erzählung verschiedener Visionen von Fratres oder Erscheinungen von Fratres vor Dritten. In diesem Abschnitt wird jede Vision vom Narrator kommentiert. Fragen der Doktrin, die zur Sprache kommen, sind z.B. die Gefahr, dass eine Vision von Teufeln verursacht wird, die Beziehung der Engel zu den Menschen, die Notwendigkeit der Beichte oder das Erbarmen mit den Sündern.

\section{Editionen:}

Delorme, Ferdinand M., Dialogus de gestis sanctorum fratrum Minorum, auctore fr. Thoma de Papia, Ad Claras Aquas (Quaracchi) 1923. Kritische Edition der Handschrift Vat. Borgh. 347.

Exzerpte in:

Papebroch (van Papenbroeck), Daniel, in Acta Sanctorum Iunii II, Antwerpen 1698, 718a-723a; spätere Editionen: Acta Sanctorum Iunï II, Venezia 1742, 718-722; Acta Sanctorum Iunii III, Paris 1867, 210-214. Nur der Abschnitt 
über das Leben des Antonius von Padua, nach einer heute verlorenen Handschrift aus dem Franziskanerkloster in Ancona.

Lemmens, Leonhard, Dialogus de vitis sanctorum fratrum Minorum, Roma 1902. Text aus der vatikanischen Handschrift, aber unvollständig.

Abate, Giuseppe, „La leggenda antoniana del Dialogus nel codice de Chambéry", Miscellanea Francescana 32 (1932), 119-138. Nur das Leben des Antonius von Padua. Text der Handschrift aus Chambéry mit Varianten aus der Handschrift im Vatikan.

Abate, Giuseppe, ,,Testo della leggenda antoniana contenuta nel codice di Chambéry“", Il Santo 9 (1969), 320-324. Nur das Leben des Antonius von Padua nach der Handschrift in Chambéry.

Abate, Giuseppe, „Testo della leggenda antoniana del Dialogus secondo il codice Vaticano-Assisano", Il Santo 9 (1969), 310-320. Nur das Leben des Antonius nach der Vatikanischen Handschrift.

Gamboso, Vergilio, Vita del „Dialogus“ e „Benignitas“, Padova 1986. Kritische Edition des Abschnitts über Antonius. Text mit italienischer Übersetzung 94-229.

\section{Literatur:}

Bertazzo, Luciano (Hg.), „Vite“ e vita di Antonio di Padova. Atti del Convegno internazionale sulla agiografia antoniana, Padova 29 maggio-1 giugno 1995, Padova 1997.

Ciccarelli, Diego, Art. „Thomas von Pavia“", LMA 8 (1997), 722.

Dalarun, Jacques, „Miracolo e miracoli nell'agiografia antoniana“, in: Bertazzo, 203-239.

Leonardi, Claudio, „L'Antonio delle biografie“, in: Bertazzo, 31-43.

Schürer, Markus, Das Exemplum oder die erzählte Institution. Studien zum Beispielgebrauch bei den Dominikanern und Franziskanern des 13. Jahrhunderts, Münster u. a. 2005, hier 237-265.

\section{Überlieferung:}

Aus der Edition von Gamboso (83-86, über verlorene Handschriften 86-90):

Chambéry, BM, 4, ca. 1427-35, fol. 496-499. Exzerpte über das Leben des Antonius von Padua.

Vaticano, BAV, Borgh. 347, 13. Jh. (2. H.), fol. 1-152. Vollständig.

Hinzu kommt:

Assisi, Biblioteca storico-francescana della Chiesa Nuova, 380, 13. Jh. (Ende), fol. 1r-150r. Vollständig. Titel: Dyalogus sanctorum fratrum minorum.

Folgende Handschrift aus Mohan konnte nicht überprüft werden:

Assisi, Com. 691, 14. Jh., fol. 45r. 